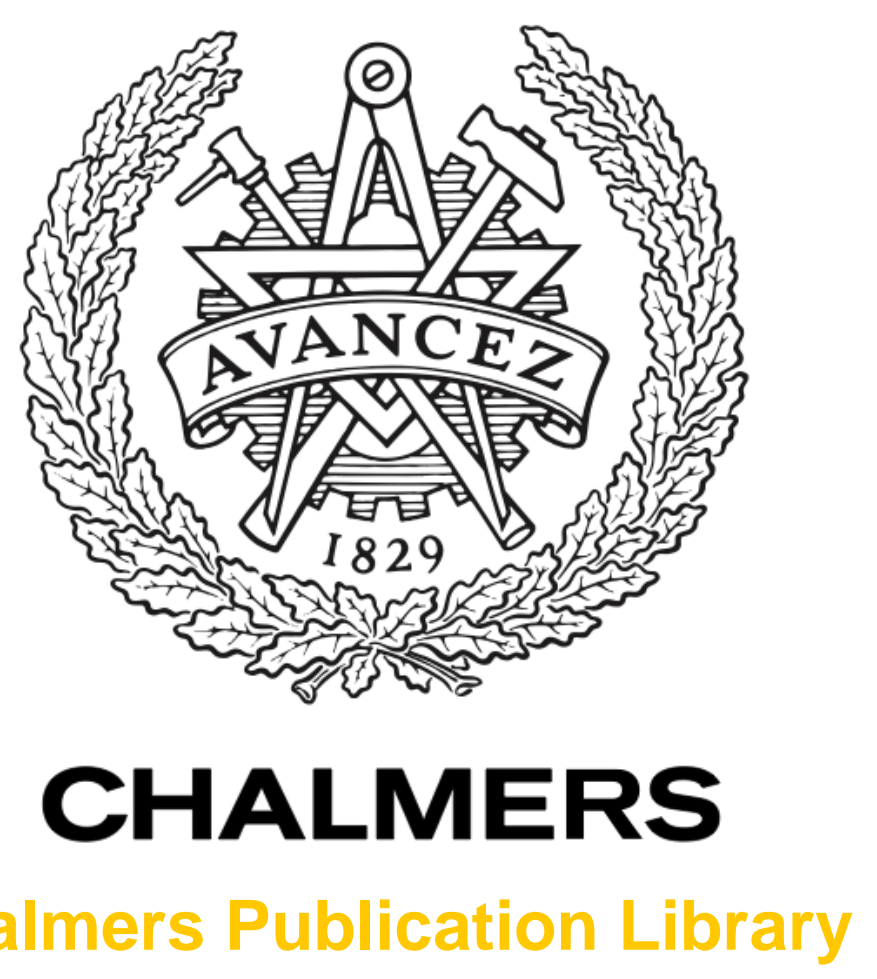

Chalmers Publication Library

Throughput Multiplexing Efficiency for MIMO Antenna Characterization

This document has been downloaded from Chalmers Publication Library (CPL). It is the author's version of a work that was accepted for publication in:

IEEE Antennas and Wireless Propagation Letters (ISSN: 1536-1225)

Citation for the published paper:

Chen, X. (2013) "Throughput Multiplexing Efficiency for MIMO Antenna Characterization". IEEE Antennas and Wireless Propagation Letters, vol. 12 pp. 1208-1211.

Downloaded from: http://publications.lib.chalmers.se/publication/184562

Notice: Changes introduced as a result of publishing processes such as copy-editing and formatting may not be reflected in this document. For a definitive version of this work, please refer to the published source. Please note that access to the published version might require a subscription.

Chalmers Publication Library (CPL) offers the possibility of retrieving research publications produced at Chalmers University of Technology. It covers all types of publications: articles, dissertations, licentiate theses, masters theses, conference papers, reports etc. Since 2006 it is the official tool for Chalmers official publication statistics. To ensure that Chalmers research results are disseminated as widely as possible, an Open Access Policy has been adopted.

The CPL service is administrated and maintained by Chalmers Library. 


\title{
Throughput Multiplexing Efficiency for MIMO Antenna Characterization
}

\author{
Xiaoming Chen
}

\begin{abstract}
The antenna-channel effects on multiple-input-multiple-output (MIMO) throughput are characterized in terms of spatial multiplexing (SM) efficiency. In order to show the antenna effects explicitly, the SM efficiency of an MIMO system (with imperfect transmit antennas) is derived analytically at high signal-to-noise ratio (SNR) regime. It is shown that correlation and power imbalance affect the throughput adversely (as expected) and independently. Although derived for imperfect transmit antennas, the close-form SM efficiency formula turns out to be accurate for $2 \times 2$ MIMO systems with imperfect receive antennas as well. It is also shown that the derived SM efficiency formula even reasonably predicts the measured SM efficiency of a Long Term Evolution (LTE) system.
\end{abstract}

Index Terms-Antennas, multiple-input-multiple-output (MIMO), spatial multiplexing (SM), throughput.

\section{INTRODUCTION}

A NTENNA effects on multiple-input-multiple-output (MIMO) systems have been characterized in various over-the-air (OTA) tests [1]-[6]. In [2] and [3], MIMO antennas are characterized by MIMO capacities calculated from measured MIMO channels. In [4] and [5], MIMO antennas are characterized by measuring the throughputs (i.e., the actual data rate) of MIMO systems. In [6], a spatial multiplexing (SM) efficiency is proposed for MIMO antenna characterization. The SM efficiency [6] is defined as the signal-to-noise ratio (SNR) degradation due to imperfect MIMO antenna (with nonzero correlations and nonunity antenna efficiencies) at certain capacity level. The SM efficiency is a convenient metric for antenna engineers to design MIMO antennas with the aim of optimizing MIMO capacity [6]. Nevertheless, in practice the capacity is usually not achievable (due to, e.g., non-Gaussian input and finite set of modulation and coding schemes in practical systems), which motivates the extension of the SM efficiency to characterizing throughput degradation due to imperfect MIMO antennas. For distinction, we refer to the original SM efficiency [6] as capacity SM efficiency and the SM efficiency in this letter as throughput SM efficiency. Without specifications hereafter, SM efficiency means throughput SM efficiency.

Manuscript received August 26, 2013; accepted September 18, 2013. Date of publication September 20, 2013; date of current version October 02, 2013.

The author is with the Department of Signals and Systems, Chalmers University of Technology, Gothenburg 412 96, Sweden (e-mail: xiaoming.chen@chalmers.se).

Color versions of one or more of the figures in this letter are available online at http://ieeexplore.ieee.org.

Digital Object Identifier 10.1109/LAWP.2013.2282952
A spatial multiplexing throughput model that shows good agreement with the measurement was developed in [4], which also contains a brief discussion of the SM efficiency. In this letter, we assume an open-loop MIMO system (where the transmitter sends independent streams with equal power) in that closed-loop MIMO OTA testing system is not available to date [1]. For analytical tractability, Rayleigh flat fading channel and zero forcing (ZF) equalizer are assumed. For a Long Term Evolution (LTE) MIMO system working in a frequency-selective fading channel, the model in this letter is valid for one subcarrier of the orthogonal frequency-division multiplexing (OFDM) system, and it can be easily extended to model the overall throughput by coherently combining independent subcarriers. The SM efficiency is analytically derived and validated by numerical simulations. Although the throughput model [4] significantly simplifies the simulation by avoiding the time-consuming implementation of Hybrid Automatic Repeat reQuest (HARQ) in LTE systems, to numerically determine the SM efficiency, one still need to perform inverse mapping at certain throughput level (cf. Section II); and this procedure needs to be repeated for each correlation or power imbalance value. Hence, the analytical formula provides a convenient alternative for calculating the SM efficiency. In addition, the analytical formula shows that the adverse effects of power imbalance and correlation on throughput are independent (separable). This finding implies that antenna engineers can optimize antenna efficiencies and correlation independently for throughput enhancement. Finally, the throughput SM efficiency is compared to the capacity SM efficiency as well as that of an LTE device measured in a reverberation chamber. Results show that the analytical throughput SM efficiency agrees reasonably well with the measurement of an LTE system, whereas the capacity SM efficiency tends to underestimate the impairment caused by imperfect antennas.

\section{Spatial Multiplexing EfFiciency}

The spatial multiplexing throughput model has been presented in [4]. According to the model, the average block error rate (BLER) in a fading channel can be approximated as

$$
\overline{P_{e}}(\bar{\gamma})=\int_{0}^{\gamma_{\mathrm{th}}} f(\gamma ; \bar{\gamma}) d \gamma=F\left(\gamma_{\mathrm{th}} ; \bar{\gamma}\right)
$$

where $\gamma$ is the instantaneous SNR, $\bar{\gamma}$ represents the average $\gamma, \gamma_{\text {th }}$ is the threshold value, and $f$ and $F$ denote the probability density function (PDF) and cumulative distribution function (CDF) of $\gamma$ for a given $\bar{\gamma}$, respectively. The approximation (1) can be justified by the outage theorems [7], which state that, 
with powerful coding, the average BLER can be well approximated by the outage probability of the fading channel. As a result, we will use CDF and BLER interchangeably in this letter.

In a flat fading channel (or at one OFDM subcarrier), the MIMO system can be modeled as

$$
\mathbf{y}=\mathbf{H x}+\mathbf{n}
$$

where $\mathbf{H}$ is the MIMO channel matrix, $\mathbf{x}$ and $\mathbf{y}$ are the transmitted and received signal vectors, respectively, and $\mathbf{n}$ is the noise vector with independent identically distributed (i.i.d.) Gaussian variables. The MIMO channel including the overall antenna effect can be expressed as [2], [6]

$$
\mathbf{H}=\mathbf{R}_{\mathrm{R}}^{1 / 2} \mathbf{H}_{\mathrm{w}} \mathbf{R}_{\mathrm{T}}^{1 / 2}
$$

where $\mathbf{H}_{\mathrm{w}}$ denotes the spatially white MIMO channel with i.i.d. and unit variance complex Gaussian variables, $\mathbf{R}_{T}$ and $\mathbf{R}_{\mathbf{R}}$ are transmit and receive covariance matrices, respectively, and $\mathbf{R}_{\mathrm{T}}^{1 / 2}$ is the Hermitian square root of $\mathbf{R}_{\mathrm{T}}$, which is defined as [2]

$$
\mathbf{R}_{\mathrm{T}}=\boldsymbol{\Xi}_{\mathrm{T}} \circ \boldsymbol{\Phi}_{\mathrm{T}}
$$

where $\boldsymbol{\Xi}_{\mathrm{T}}=\sqrt{\mathbf{e}} \sqrt{\mathbf{e}}^{\mathrm{T}}$ with $\mathbf{e}$ denoting a column vector consisting antenna efficiencies of the transmit antennas, the superscript ${ }^{\mathrm{T}}$ is the transpose operator, $\sqrt{ }$ is the element-wise square root, o denotes element-wise product, and the matrix $\boldsymbol{\Phi}_{\mathrm{T}}$ consists of correlation coefficients between transmit antennas. $\mathbf{R}_{R}$ can be calculated similarly as $\mathbf{R}_{\mathrm{T}}$. For analytical tractability, we first consider imperfect transmit antennas and ideal receive antennas (i.e., $\mathbf{H}=\mathbf{H}_{\mathrm{w}} \mathbf{R}_{\mathrm{T}}^{1 / 2}$ with $\mathbf{R}_{\mathrm{R}}$ reducing to an identity matrix).

In an open-loop MIMO system, there will be multistream interference. In this letter, we use a ZF receiver [8] to cope with the interference. The ZF SNR of the $i$ th stream can be easily derived as

$$
\gamma_{i}=\bar{\gamma}_{0} /\left[\left(\mathbf{H}^{H} \mathbf{H}\right)^{-1}\right]_{i, i}
$$

where $\bar{\gamma}_{0}=\mathrm{E}\left[\left|x_{i}\right|^{2}\right] / \mathrm{E}\left[\left|n_{i}\right|^{2}\right]$ with $x_{i}$ and $n_{i}$ denoting the $i$ th elements of $\mathbf{x}$ and $\mathbf{n}$, respectively, and $[\mathbf{X}]_{i, i}$ denotes the $i$ th diagonal element of the matrix $\mathbf{X}$.

The SNR distribution of the ZF equalizer accounting for transmit correlation has been derived in [9]. Following the same lines, the ZF SNR distribution accounting for both transmit correlation and power imbalance (due to different antenna efficiencies) can be derived in a straightforward way as

$$
f\left(\gamma_{i} ; \bar{\gamma}_{0}, \mu_{i}\right)=\frac{\left(\gamma_{i} /\left(\mu_{i} \bar{\gamma}_{0}\right)\right)^{N_{\mathrm{R}}-N_{\mathrm{T}}}}{\mu_{i} \bar{\gamma}_{0} \Gamma\left(N_{\mathrm{R}}-N_{\mathrm{T}}+1\right)} \exp \left(-\frac{\gamma_{i}}{\mu_{i} \bar{\gamma}_{0}}\right)
$$

where $\Gamma$ is the Gamma function, and $\mu_{i}=1 /\left[\mathbf{R}_{\mathrm{T}}^{-1}\right]_{i, i}$. The average BLER of the ZF equalizer can be obtained by substituting (6) into (1)

$$
\overline{P_{e}}\left(\bar{\gamma}_{i}\right)=\Upsilon\left(N_{\mathrm{R}}-N_{\mathrm{T}}+1, \frac{\gamma_{\mathrm{th}}}{\bar{\gamma}_{i}}\right) / \Gamma\left(N_{\mathrm{R}}-N_{\mathrm{T}}+1\right)
$$

where

$$
\begin{aligned}
\Upsilon\left(N_{\mathrm{R}}-N_{\mathrm{T}}+1\right. & \left.1, \frac{\gamma_{\mathrm{th}}}{\bar{\gamma}_{i}}\right) \\
& =\int_{0}^{\gamma_{\mathrm{th}}} \frac{1}{\bar{\gamma}_{i}}\left(\frac{\gamma_{i}}{\bar{\gamma}_{i}}\right)^{N_{\mathrm{R}}-N_{\mathrm{T}}} \exp \left(-\frac{\gamma_{i}}{\bar{\gamma}_{i}}\right) d \gamma_{i}
\end{aligned}
$$

is the lower incomplete gamma function and $\bar{\gamma}_{i}=\mu_{i} \bar{\gamma}_{0}$.

The MIMO throughput can be modeled as

$$
T_{\text {put }}(\bar{\gamma})=\frac{T_{\text {put }, \text { max }}}{N_{\mathrm{T}}} \sum_{i=1}^{N_{\mathrm{T}}}\left(1-\bar{P}_{e}\left(\bar{\gamma}_{i}\right)\right)
$$

where $T_{\text {put,max }}$ denotes the maximum achievable throughput and $\bar{\gamma}=N_{\mathrm{T}} \bar{\gamma}_{0}$. Note that in this letter we define the normalized throughput $T=T_{\text {put }} / T_{\text {put,max }}$ as the relative throughput, which will be used throughout this letter.

In order to conveniently characterize the antenna effect on the throughput, we resort to SM efficiency defined as the inverse of SNR increase (or SNR degradation) in the presence of imperfect antennas in order to achieve the same throughput of an ideal fading channel (i.e., i.i.d. channel with ideal antennas). Mathematically, it can be expressed as

$$
e_{\text {mux }}=\left.\frac{T_{0}^{-1}(\bar{\gamma})}{T^{-1}(\bar{\gamma})}\right|_{95 \%}
$$

where $T^{-1}$ denotes functional inversion of the relative throughput $T$, and $T_{0}$ represents the relative throughput for the ideal fading channel. The choice of evaluating the SM efficiency at $95 \%$ throughput level is motivated by the fact that $95 \%$ throughput is usually of interest [1]. The corresponding results are valid at least at high throughput levels. Note that the throughput model is valid for MIMO-OFDM systems with HARQ (such as LTE systems) and fixed modulation and coding scheme (MCS). The fixed MCS is a common setting for OTA tests, because of which only the high throughput level is relevant in that, at low throughput levels, a lower MCS will be used.

In order to show the effects of transmit (complex) correlation coefficient $\rho$ and antenna efficiencies $e_{i}(i=1,2)$ on throughput explicitly, we assume $N_{\mathrm{R}} \times 2$ MIMO system with $N_{\mathrm{R}}$ ideal receive antennas. The choice of two transmit antennas enables us to express the SM efficiency in terms of correlation and antenna efficiencies; it can also be motivated by the fact that most of the current LTE systems support up to two independent streams [1]. In this case, $\mu_{i}=1 /\left[\mathbf{R}_{\mathrm{T}}^{-1}\right]_{i, i}$ becomes $e_{i}\left(1-|\rho|^{2}\right)$. At the high-SNR regime, the BLER of the $i$ th stream (7) becomes

$$
\frac{\Upsilon\left(N_{\mathrm{R}}-1, \frac{\gamma_{\text {th }}}{\bar{\gamma}_{0} e_{i}\left(1-|\rho|^{2}\right)}\right)}{\left(N_{\mathrm{R}}-2\right) !} \approx \frac{\left(\frac{\gamma_{\text {th }}}{\bar{\gamma}_{0} e_{i}\left(1-|\rho|^{2}\right)}\right)^{N_{\mathrm{R}}-1}}{\left(N_{\mathrm{R}}-1\right) !}
$$

where ! denotes the factorial operator and the approximation is accurate high throughput level (e.g., 95\% throughput level). Combining (10), (11), and (9), the SM efficiency becomes

$$
e_{\operatorname{mux}}=2\left(1 / e_{1}^{N_{\mathrm{R}}-1}+1 / e_{2}^{N_{\mathrm{R}}-1}\right)^{-1 /\left(N_{\mathrm{R}}-1\right)}\left(1-|\rho|^{2}\right) .
$$




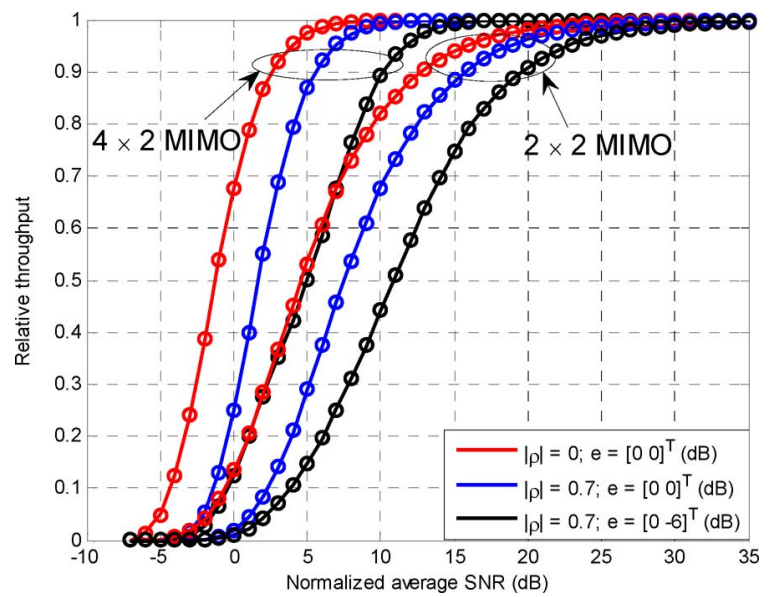

Fig. 1. Relative throughputs of $2 \times 2$ and $4 \times 2$ MIMO systems for the transmit semi-correlation case. The solid curves correspond to empirical CDFs. The circles correspond to the theoretical CDF (10).

Note that the capacity SM efficiency was originally proposed in [6] as the SNR degradation in presence of imperfect antennas at certain capacity level. To facilitate the comparison, we assume a $2 \times 2$ MIMO system. In this case, the SM efficiency (16) reduces to

$$
e_{\text {mux }}=2\left(1 / e_{1}+1 / e_{2}\right)^{-1}\left(1-|\rho|^{2}\right)
$$

and the capacity SM efficiency (at high SNR) is [6]

$$
\eta_{\text {mux }}=\sqrt{e_{1} e_{2}\left(1-|\rho|^{2}\right)} .
$$

Comparing (13) and (14), it can be seen that the effects of power imbalance and correlation are independent (separable) and that they are more profound for $e_{\operatorname{mux}}$ than for $\eta_{\operatorname{mux}}$.

\section{Simulation AND MEasurement}

In this section, we will study the antenna effects at transmitter as well as at receiver. We hereafter refer to the case of imperfect transmit antennas and ideal receive antennas (i.e., $\mathbf{H}=$ $\mathbf{H}_{\mathrm{w}} \mathbf{R}_{\mathrm{T}}^{1 / 2}$ ) as transmit semi-correlation and the case of imperfect receive antennas and ideal transmit antennas (i.e., $\mathbf{H}=$ $\mathbf{R}_{\mathrm{R}}^{1 / 2} \mathbf{H}_{\mathrm{w}}$ ) as receive semi-correlation.

In order to obtain the empirical CDF (or the average BLER) of the SNR, we sample 10000 snapshots of the simulated channel and calculate 10000 instantaneous SNR accordingly. The throughput is then calculated using (9).

Fig. 1 shows the calculated throughputs for $2 \times 2$ and $4 \times 2$ MIMO systems for the transmit semi-correlation case. The solid curves are obtained from empirical CDF; the circles are obtained using the theoretical CDF. The red curves represents the throughput with ideal transmit antennas; the blue curve denotes the throughput with a transmit correlation of 0.7 (in magnitude); the black curve represents the throughput with a transmit correlation of 0.7 and a power imbalance of $6 \mathrm{~dB}$, i.e., $\mathbf{e}=$ $[0-6]^{\mathrm{T}}(\mathrm{dB})$. The left group of three curves (in red, blue, and black) represents the throughputs of $4 \times 2$ MIMO systems; the right group of three curves (in red, blue, and black) represents the throughputs of $2 \times 2$ MIMO systems. It can be seen that both correlation and power imbalance tend to degrade the throughput
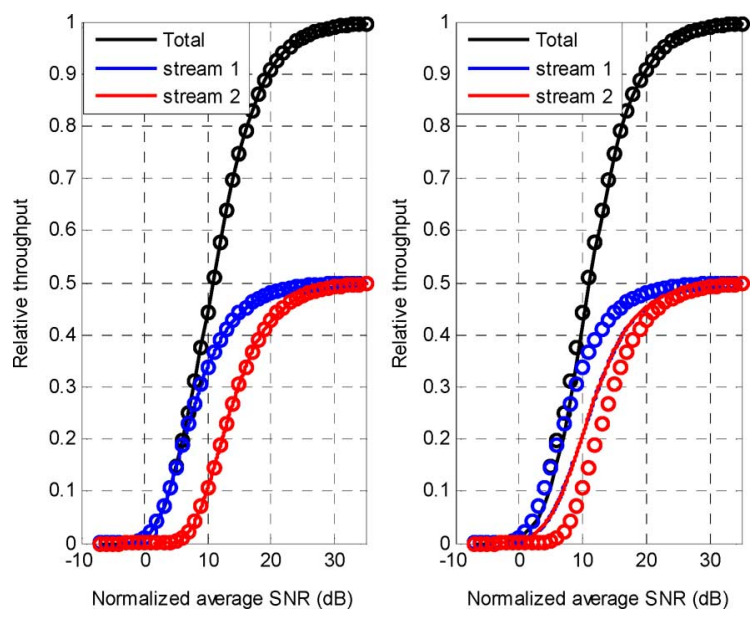

Fig. 2. Relative throughputs of a $2 \times 2$ MIMO system for (left) transmit semicorrelation case and (right) receive semi-correlation case. The solid curves correspond to empirical CDFs. The circles correspond to the theoretical CDF (10) for $\mathbf{R}_{\mathrm{T}}=\mathbf{R}_{\mathrm{R}}$. An MIMO antenna with $\mathbf{e}=[0-6]^{\mathrm{T}}(\mathrm{dB})$ and $|\rho|=0.7$.

performance and that, with more receive antennas, there are improvements in both SNR and diversity order. Note that the normalized average SNR is $\bar{\gamma} / \gamma_{\text {th }}$ (cf. Section II). The sum of the average SNRs at all the receive antennas for the $4 \times 2$ MIMO system causes a shift of the throughput curve to the left w.r.t. to the $2 \times 2$ MIMO system. This is due to the array factor [8]. The ZF diversity order is $N_{\mathrm{R}}-N_{\mathrm{T}}+1$ [8]. From $2 \times 2$ to $4 \times 2$ MIMO system, the diversity order increases by 2 . This causes a larger (steeper) slope of the throughput curve.

Note that the ZF SNR distribution (7) is derived for the transmit semi-correlation case, i.e., $\mathbf{H}=\mathbf{H}_{\mathrm{w}} \mathbf{R}_{\mathrm{T}}^{1 / 2}$. This unique channel structure makes $\mathbf{H}^{H} \mathbf{H}$ in (8) a Wishart matrix [10] whose distribution is well known, which in turn enables the derivation of the ZF SNR distribution [9]. Although $\mathbf{H}^{H} \mathbf{H}$ with $\mathbf{H}=\mathbf{R}_{\mathrm{R}}^{1 / 2} \mathbf{H}_{\mathrm{w}}$ shares the same eigenvalue distribution as the Wishart matrix (when $\mathbf{R}_{\mathrm{R}}=\mathbf{R}_{\mathrm{T}}$ ), its distribution is unknown to date. Therefore, so far there is no exact expression for the ZF SNR distribution for the receive semi-correlation case. Fig. 2 compares the theoretical throughput (derived for the transmit semi-correlation case) to the simulated throughputs for both transmit and receive semi-correlation cases. It is shown that, although (7) is not a good approximation for BLER of an individual stream for the latter case, the throughput (9) [together with (7)] well predicts that of the latter case. This means that the SM efficiency (13) derived for the transmit semi-correlation case is also a good approximation for the receive semi-correlation case.

In order to numerically determine the SM efficiency, we repeat the simulations for different power imbalances and correlations, separately. Figs. 3 and 4 show the SM efficiency of a two-element MIMO antenna as a function of correlation and power imbalance in $4 \times 2$ and $2 \times 2$ MIMO systems, respectively. The empirical SM efficiency obtained from simulation is shown as a solid blue curve. The theoretical SM efficiency $e_{\text {mux }}$ is shown as red circles. For comparison, the theoretical capacity SM efficiency $\eta_{\text {mux }}$ is also shown as a black dotted curve in Fig. 4. It can be seen that the derived SM efficiency $e_{\text {mux }}$ agrees well with simulations and that the capacity SM efficiency 

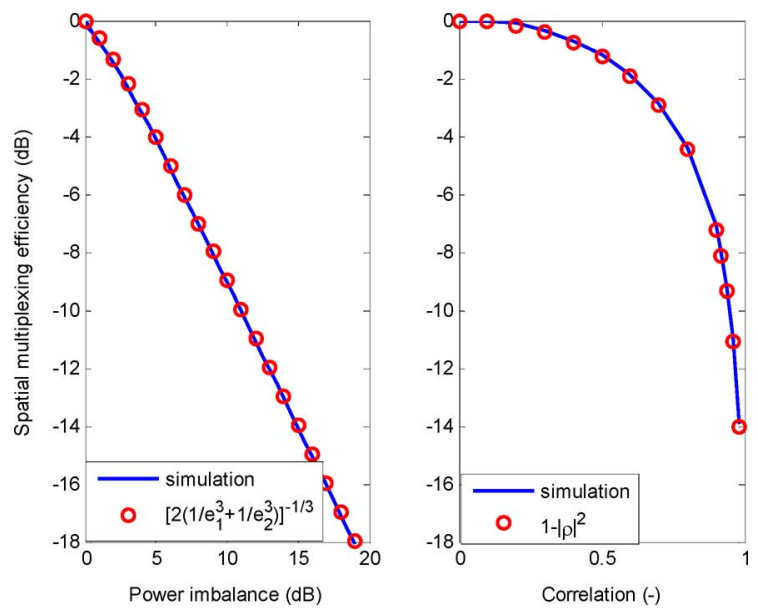

Fig. 3. SM efficiencies for $4 \times 2$ MIMO systems with empirical values obtained from simulations (solid curve) and theoretical value (circles) from (16).
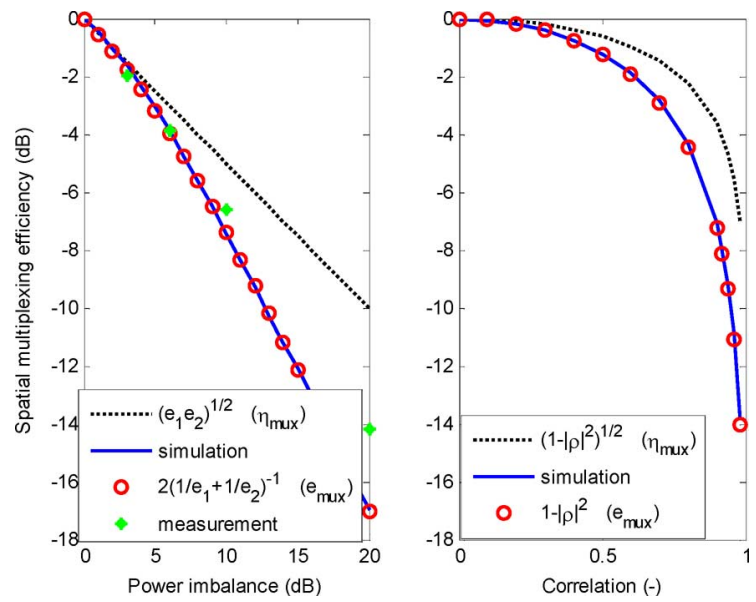

Fig. 4. SM efficiencies of $2 \times 2$ MIMO systems with empirical values obtained from simulations (solid curve) and theoretical value (circles) from (17). The theoretical capacity SM efficiencies (dotted curve) obtained from (18) and the measured throughput SM efficiencies (asterisks) are also shown (at 3-, 6-, 10-, and $20-\mathrm{dB}$ power imbalances) for comparison.

$\eta_{\text {mux }}$ is higher than $e_{\text {mux }}$, meaning the antenna effects have less influence on the MIMO capacity. The latter observation is intuitive: In order to achieve capacity, a more advanced receiver (e.g., maximum likelihood (ML) receiver [8]) is needed; and this optimal yet impractical ML receiver is able to further mitigate the antenna effect. It has been shown that the throughput SM efficiency derived in this letter is more accurate in characterizing LTE MIMO antennas. Note that the derived throughput SM efficiency is for the linear ZF equalizer. For a system (other than the LTE system) where the close-to-ML decoder (e.g., spherical decoder [8]) is implemented, the capacity SM efficiency will be more suitable.

In order to compare the theoretical SM efficiency to the measured one of an LTE system, throughput measurements were conducted in a reverberation chamber [4] (where the measurement setup is described). For each power imbalance case (i.e., $0,3,6,10$, and $20 \mathrm{~dB}$ ), we sweep the transmit power at the base station such that an average $95 \%$ throughput is achieved. The measured SM efficiencies are shown as asterisks in Fig. 4. It can be seen that the measured SM efficiency is very close to the theoretical SM efficiency $e_{\operatorname{mux}}$ up to $10-\mathrm{dB}$ power imbalance.
Note that a $20-\mathrm{dB}$ power imbalance may be rare in practice; we included it here for the verification of the derived SM efficiency and to show the 3-dB discrepancy of the derived SM efficiency with the measurement for the $20-\mathrm{dB}$ power imbalance case. Also note that the measurements (cf. [4]) were conducted with $10-\mathrm{MHz}$ system bandwidth and 3-MHz coherence bandwidth (the latter is a common RC-OTA setup [11]). It has been checked numerically that, by taking into account the $2 \sim 3$ order of frequency diversity (cf. Section II), there is very little change in the simulated SM efficiency.

\section{CONCLUSION}

In order to show the antenna effect on the MIMO throughput explicitly, the throughput SM efficiency is proposed. The theoretical ZF SNR distribution is obtained, based on which a closed-form expression of the SM efficiency is derived for $N_{\mathrm{R}} \times 2$ MIMO systems for the transmit semi-correlation case. It is found that the throughput for the receive semi-correlation case can be well approximated by the theoretical throughput for the transmit semi-correlation case. Therefore, the derived SM efficiency is valid for both semi-correlation cases. Good agreements are observed between the theoretical SM efficiency and the simulated one. It is shown from the derived SM efficiency that the effects of correlation and power imbalance on the throughput are separable. It is also shown that the measured SM efficiencies of $2 \times 2$ LTE MIMO systems under different power imbalance conditions agree well with the theoretical throughput SM efficiency up to $10-\mathrm{dB}$ power imbalance.

\section{REFERENCES}

[1] A. A. Glazunov, V. M. Kolmonen, and T. Laitinen, "MIMO overthe-air testing," in LTE-Advanced and Next Generation Wireless Networks: Channel Modelling and Propagation. Hoboken, NJ, USA: Wiley, pp. 411-441

[2] X. Chen, P.-S. Kildal, J. Carlsson, and J. Yang, "MRC diversity and MIMO capacity evaluations of multi-port antennas using reverberation chamber and anechoic chamber," IEEE Trans. Antennas Propag., vol. 61, no. 2, pp. 917-926, Feb. 2013.

[3] J. F. Valdes, M. A. Garcia-Fernandez, A. M. Martinez-Gonzalez, and D. A. Sanchez-Hernandez, "The influence of efficiency on receive diversity and MIMO capacity for Rayleigh-fading channels," IEEE Trans. Antennas Propag., vol. 56, no. 5, pp. 1444-1450, May 2008.

[4] X. Chen, P.-S. Kildal, and M. Gustafsson, "Characterization of implemented algorithm for MIMO spatial multiplexing in reverberation chamber," IEEE Trans. Antennas Propag., vol. 61, no. 8, pp. 4400-4404, Aug. 2013.

[5] R. Recanatini, F. Moglie, and V. M. Primiani, "Performance and immunity evaluation of complete WLAN systems in a large reverberation chamber," IEEE Trans. Electromagn. Compat., 2013, to be published.

[6] R. Tian, B. K. Lau, and Z. Ying, "Multiplexing efficiency of MIMO antennas," IEEE Antennas Wireless Propag. Lett., vol. 10, pp. 183-186, 2011.

[7] N. Prasad and M. K. Varanasi, "Outage theorems for MIMO block-fading channels," IEEE Trans. Inf. Theory, vol. 58, no. 7, pp. 2159-2168, Jul. 2006

[8] A. Paulraj, R. Nabar, and D. Gore, Introduction to Space-Time Wireless Communication. Cambridge, U.K.: Cambridge Univ. Press, 2003.

[9] D. Gore, R. W. Heath, and A. Paulraj, "On performance of the zero forcing receiver in presence of transmit correlation," in Proc. IEEE ISIT, Lausanne, Switzerland, Jun. 30-Jul. 5 2002, p. 159.

[10] J. Wishart, "The generalized product moment distribution in samples from a normal multivariate population," Biometrika, vol. 20, pp. 32-52, Dec. 1928.

[11] E. Genender, C. L. Holloway, K. A. Remley, J. M. Ladbury, G. Koepke, and H. Garbe, "Simulating the multipath channel with a reverberation chamber: Application to bit error rate measurements," IEEE Trans. Electromagn. Compat., vol. 52, no. 4, pp. 766-777, Nov. 2010. 